\title{
Fast Detection of Venous Air Embolism in Doppler Heart Sound Using the Wavelet Transform
}

\author{
Brent C. B. Chan, Student Member, IEEE, Francis H. Y. Chan,* Member, IEEE, \\ F. K. Lam, Ping-Wing Lui, and Paul W. F. Poon
}

\begin{abstract}
The introduction of air bubbles into the systemic circulation can result in significant morbidity. Real-time monitoring of continuous heart sound in patients detected by precordial Doppler ultrasound is, thus, vital for early detection of venous air embolism (VAE) during surgery. In this study, the multiscale feature of wavelet transforms (WT's) is exploited to examine the embolic Doppler heart sound (DHS) during intravenous air injections in dogs. As both humans and dogs share similar physiological conditions, our methods and results for dogs are expected to be applicable to humans. The WT of DHS at scale $2^{j}(j=1,2)$ selectively magnified the power of embolic, but not the normal, heart sound. Statistically, the enhanced embolic power was found to be sensitive $(P<0.01$ at $0.01 \mathrm{ml}$ of injected air) and correlated significantly $(P<0.0005, r=0.83)$ with the volume of injected air from 0.01 to $0.10 \mathrm{ml}$. A fast detection algorithm of $O(N)$ complexity with unit complexity constant for VAE was developed (processing speed $=8 \mathrm{~ms}$ per heartbeat), which confirmed the feasibility of real-time processing for both humans and dogs.
\end{abstract}

Index Terms - Precordial doppler ultrasound, venous air embolism, wavelet transform.

\section{INTRODUCTION}

V ENOUS AIR embolism (VAE) occurs intermittently during surgery when the incision wounds lie above the heart. The central venous pressure in the cut vessels being lower than the atmospheric pressure causes air to be sucked to the circulatory system. As VAE is life-threatening, real-time monitoring of the continuous heart sound is essential for early and prompt detection of VAE and for issuing instant warning to the anaesthetist to insure proper clinical treatment.

Ultrasonic precordial Doppler monitoring is commonly used for the detection of VAE [1], [2]. This method is superior to other methods, especially in terms of sensitivity and noninvasive nature [3]. The audio Doppler heart sound (DHS)

Manuscript received September 28, 1995; revised December 2, 1996. Asterisk indicates corresponding author. This work was supported in part by The University of Hong Kong under research grants, and in part by the National Science Council under Grant NSC85-2331-B006-064.

B. C. B. Chan is with the Department of Electrical and Electronic Engineering, The University of Hong Kong, Hong Kong.

*F. H. Y. Chan is with the Department of Electrical and Electronic Engineering, The University of Hong Kong, Pokfulam Road, Hong Kong (e-mail: fhychan@eee.hku.hk).

F. K. Lam is with the Department of Electrical and Electronic Engineering, The University of Hong Kong, Hong Kong.

P. W. Lui is with the Department of Anesthesiology, Veterans General Hospital-Taipei, Taipei, Taiwan.

P. W. F. Poon is with the Department of Physiology, School of Medicine, National Cheng Kung University, Tainan, Taiwan.

Publisher Item Identifier S 0018-9294(97)02221-0. can be obtained simply by placing the Doppler ultrasonic flow transducer over the chest of the patient (as compared with a common invasive method known as transesophageal echocardiography (TEE) [4] which causes substantial discomfort by requiring the patient to swallow an ultrasonic probe of considerable size together with the attached cables). A disadvantage of the Doppler method is that it requires the constant attention of the anaesthetist to detect subtle changes in the DHS. In addition, quantitative information on the volume of air entering circulation cannot be provided simply by listening to the audio DHS. As anaesthetists usually act only when a clinically significant volume (above $1 \mathrm{ml}$ ) of air embolism is present [5], such quantitative information is crucial for them to prescribe suitable clinical treatment.

In order to study both the qualitative and quantitative properties of the DHS during VAE, we have to inject a known volume of air into the vessels and monitor the changes in the DHS. However, it is unethical to try this experiment in humans as VAE is a potentially fatal anaesthetic complication. Thus, we conducted our experiment on dogs as most of the physiological conditions of dogs are quite similar to those of humans. Since the heart rate of dogs $(180 / \mathrm{min})$ is much higher than that of humans $(70 / \mathrm{min})$, if we can achieve real-time monitoring of the DHS in dogs, we could be confident that the same technique may also be applied to humans.

We investigated the spectral characteristics of the embolic heart sound (EHS) in anaesthetized dogs using a timefrequency method [6]. We showed that while the normal heart sound occupies the frequency band below $400 \mathrm{~Hz}$, EHS occupies an additional higher-frequency band from 400 to $1200 \mathrm{~Hz}$. With a single cutoff frequency estimated by the percentile-andthreshold crossing method [7], we established a method for quantitative analysis of embolic air during VAE. Although the high-frequency components increased significantly $(P<0.05$ by Student's $t$-test [8]) at volumes as low as $0.05 \mathrm{ml}$ of embolic air, there was much scattering in the relationship $(r=0.46$ on linear regression [8]). Moreover, the time-frequency method used was not efficient enough for real-time computation.

As part of a continuing effort to develop a real-time monitoring system for VAE [6], [9], we used an improved technique in this study that involves the wavelet transform (WT) [10]-[12]. WT is a promising technique for time-scale (or time-frequency) analysis which decomposes a signal into components that appear at different scales. It is closely related to multiscale edge detection methods [13] which can reveal points of sharp variations in transient signals such as the EHS. 
This feature can thus be used to analyze the high-frequency components of the EHS. We also utilized some features of a particular class of dyadic wavelets and its fast algorithm [13] so as to further improve the data-processing speed for real-time application. Consequently, fast detection of VAE and accurate estimation of the volume of embolic air could be attained. To confirm the superiority of the WT method, we compare the present results with those obtained by our previous method on the same set of experimental data.

\section{WAVELET TRANSFORM}

\section{A. General Properties}

$Z$ and $R$ denote the set of integers and real numbers, respectively. $L^{2}(R)$ denotes the Hilbert space of measurable, square-integrable 1-D functions. The WT is a linear operation which decomposes a signal into components that appear at different scales. A wavelet is a function $\psi(x) \in L^{2}(R)$ whose average is zero. The WT of a signal is computed by convolving the signal with a dilated wavelet. For thorough descriptions of the WT, the reader is referred to the work of Meyer [11] and Daubechies [12]. The WT of a signal $f(x)$ at scale $s$ and position $x$ is given by the convolution product

$$
W_{s} f(x)=f^{*} \psi_{s}(x)=\int_{-\infty}^{+\infty} f(t) \psi_{s}(x-t) d t
$$

where

$$
\psi_{s}(x)=\frac{1}{s} \psi\left(\frac{x}{s}\right)
$$

The support of $\psi_{s}(x)$ decreases as the scale $s$ decreases such that the WT of $f(x)$ (i.e., $W_{s} f(x)$ ) is sensitive to finer details. Hence, the scale $s$ characterizes the size and regularity of the signal features extracted by the WT.

\section{B. Dyadic Wavelet Transform}

The WT depends on two parameters, $s$ and $x$, that vary continuously over $R$. These parameters must be discretized for practical applications. For a particular class of wavelets which allows fast numerical implementation (to be discussed in the following sections), the scale parameter can be sampled along the dyadic sequence $\left(2^{j}\right)_{j \in Z}$ without modifying the overall properties of the transform. The WT at scale $2^{j}$ is given by

$$
W_{2^{j}} f(x)=f^{*} \psi_{2^{j}}(x) .
$$

The Fourier transform $(\mathcal{F}\{\cdot\})$ of $W_{2^{j}} f(x)$ is

$$
\mathcal{F}\left\{W_{2^{j}} f(x)\right\}=F(\omega) \Psi\left(2^{j} \omega\right)
$$

where $F(\omega)$ and $\Psi(\omega)$ represent the Fourier transform of $f(x)$ and $\psi(x)$, respectively. By imposing that

$$
\sum_{j=-\infty}^{+\infty}\left|\Psi\left(2^{j} \omega\right)\right|^{2}=1
$$

we ensure that the whole frequency axis is covered by the dilation of $\Psi(\omega)$ by $\left(2^{j}\right)_{j \in Z}$. Any wavelet satisfying (5)

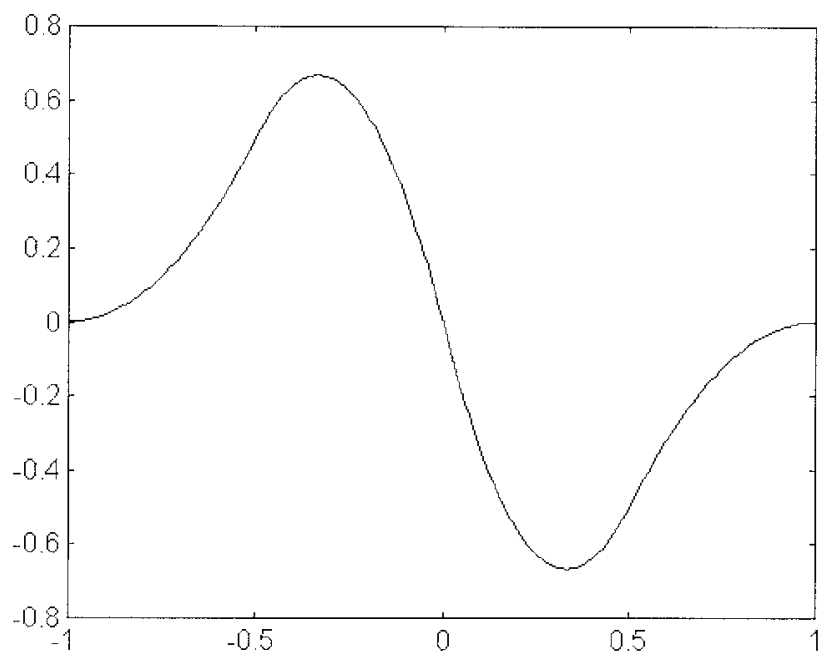

\begin{tabular}{|c|c|c|c|c|}
\hline$k$ & -1 & 0 & 1 & 2 \\
\hline$h_{k}$ & 0.125 & 0.375 & 0.375 & 0.125 \\
\hline$g_{k}$ & & -2.0 & 2.0 & \\
\hline
\end{tabular}

Fig. 1. The wavelet used in the present study is a quadratic spline of compact support which is continuously differentiable.

TABLE I

Coefficients in the Finite Impulse Responses of Filters $H$ and $G$

is called a dyadic wavelet. We also call as dyadic WT the sequence of functions

$$
W f=\left(W_{2^{j}} f(x)\right)_{j \in Z} .
$$

$W$ is then called the dyadic WT operator.

\section{A Particular Class of Wavelets}

We define a class of wavelets which can be used for fast implementation of a discrete algorithm [13]. We first define a smoothing function $\phi(x)$ whose Fourier transform, $\Phi(\omega)$, can be written as an infinite product

$$
\Phi(\omega)=e^{-i w \omega} \prod_{p=1}^{+\infty} H\left(2^{-p \omega}\right)
$$

where $H(\omega)$ is a $2 \pi$ periodic differentiable function such that

$$
|H(\omega)|^{2}+|H(\omega+\pi)|^{2} \leq 1 \quad \text { and } \quad|H(0)|=1 .
$$

The parameter $w$ is the sampling shift which arises from the discretization of the dyadic WT. It is adjusted so that $\phi(x)$ is symmetrical with respect to zero. Equation (7) implies that

$$
\Phi(2 \omega)=e^{-i w \omega} H(\omega) \Phi(\omega) .
$$

We next define a wavelet $\psi(x)$ whose Fourier transform, $\Psi(\omega)$, is given by

$$
\Psi(2 \omega)=e^{-i w \omega} G(\omega) \Phi(\omega)
$$

where $G(\omega)$ is a $2 \pi$ periodic function.

We want a wavelet, $\psi(x)$, equal to the first-order derivative of a smoothing function. This implies that $\Psi(\omega)$ must have a 


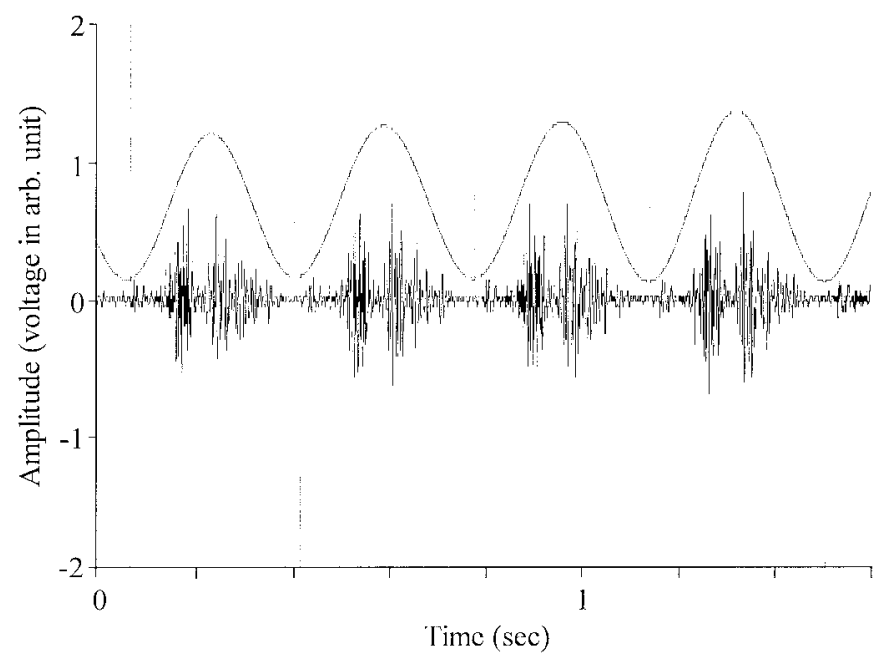

Fig. 2. Temporal partition of the DHS: the original DHS signal (lower tracing) is displayed together with the signal envelope obtained by lowpass filtering the DHS (upper tracing). The local minima of the envelope (indicated by vertical lines) represent the boundaries of individual cardiac cycles or heartbeats.

zero of order one at $\omega=0$. Since $|\Phi(0)|=1$, (10) yields that $G(\omega)$ must have a zero of order one at $\omega=0$. We choose $H(\omega)$ in order to obtain a wavelet, $\psi(x)$, which is antisymmetrical, as regular as possible, and has compact support. A family of $2 \pi$ periodic functions $H(\omega)$ and $G(\omega)$ which satisfy these constraints is given by

$$
\begin{aligned}
& H(\omega)=e^{i \omega / 2}\left(\cos \frac{\omega}{2}\right)^{2 n+1} \\
& G(\omega)=4 i e^{i \omega / 2}\left(\sin \frac{\omega}{2}\right) .
\end{aligned}
$$

From (7) and (10), we derive

$$
\begin{aligned}
& \Phi(\omega)=\left(\frac{\sin (\omega / 2)}{\omega / 2}\right)^{2 n+1} \\
& \Psi(\omega)=i \omega\left(\frac{\sin (\omega / 4)}{\omega / 4}\right)^{2 n+2} .
\end{aligned}
$$

In the present study, we chose $2 n+1=3$ and the corresponding wavelet, $\psi(x)$, is shown in Fig. 1. In order to have a wavelet antisymmetrical with respect to zero and $\phi(x)$ symmetrical with respect to zero, the shifting constant $w$ of (9) is equal to $1 / 2$. The $2 \pi$ periodic functions $H(\omega)$ and $G(\omega)$ can be viewed as the transfer functions of discrete filters with finite impulse responses. The corresponding impulse responses $h_{k}$ and $g_{k}$ are given in Table I.

\section{Fast Wavelet Algorithm}

Let $S_{2^{j}}$ be a smoothing operator at scale $2^{j}$, which is the counterpart of $W_{2^{j}}$, defined by

$$
S_{2^{j}} f(x)=f^{*} \phi_{2^{j}}(x) .
$$

For normalization purposes, our original DHS signal is represented by $S_{2^{0}} f(x)$. Based on the properties of the wavelet $\psi(x)$ described in the previous section, we can compute the
WT of the DHS signal using the following algorithm:

$$
\begin{aligned}
S_{2^{j}} f(x) & =\sum_{k \in Z} h_{k} S_{2^{j-1}} f\left(x-2^{j-1} k\right) \\
W_{2^{j}} f(x) & =\sum_{k \in Z} g_{k} S_{2^{j-1}} f\left(x-2^{j-1} k\right) .
\end{aligned}
$$

From (16) and (17), the WT can be viewed as successive filtering of the DHS signal, as the Fourier transform of the WT (17) is

$$
\begin{array}{lr}
G(\omega) F(\omega) \Phi(\omega), & j=1 ; \\
G(2 \omega) H(\omega) F(\omega) \Phi(\omega), & j=2 \\
G\left(2^{j-1} \omega\right) H\left(2^{j-2} \omega\right) \cdots H(\omega) F(\omega) \Phi(\omega), & j>2
\end{array}
$$

where $F(\omega) \Phi(\omega)$ is the Fourier transform of the original DHS signal. The WT of DHS at successive scales $\left(2^{j}, j>0\right)$ is, thus, equivalent to bandpass filtering at successively narrower bandwidths so that finer details of the DHS can be explored.

\section{EXPERIMENTAL METHODS}

\section{A. Data Collection}

Our VAE experiment on dogs was approved by the Animal Research Committee of the Veterans General Hospital, Taipei, Taiwan. Four anaesthetized dogs weighing 10-12 kg were placed in the supine position for intravenous air injections via the right external jugular vein. The Doppler ultrasonic flow transducer (Model P81 (2.4 MHz), Versatone Doppler Model D8, MedaSonics, Fremont, CA) was placed over the shaved skin at the right lateral border of the sternum using a generous quantity of coupling gel (Aquasonic 100, Parker Laboratories, Inc., Orange, NJ) and secured around the chest by Micropore tapes. We followed ascending volume steps $(0.01,0.02,0.05$, and $0.10 \mathrm{ml}$ ) with a 5 -min interval between injections. At such low volume of air injection, premature ventricular contractions (PVC) or other forms of arrhythmia were not detected as judged by an experienced anaesthetist. The audio output of the transducer was recorded first on a tape recorder (WMD6C, Sony Corp., Tokyo, Japan) and later digitized at $4 \mathrm{kHz}$, 12-b resolution through an analog and digital interface board (DT-2801A, Data Translation, Marlboro, MA) in an IBM PC (80486 DX2-66). The overall frequency response of the recording system was flat $( \pm 3 \mathrm{~dB})$ from $20 \mathrm{~Hz}$ to $18 \mathrm{kHz}$. We digitized an 8-s period of the DHS signal for each air injection with the embolic air appearing roughly at the middle of the time segment. The period of DHS with no previous injection of air was taken as the control. A total of 12 datasets were collected from three identical series of air injections on each of the four animals.

\section{B. Data Analysis}

For systematic analysis, the DHS signal was logically partitioned into a sequence of individual heartbeats. We adopted a lowpass filtering approach since the overall shape of the DHS is very similar to that of an amplitude modulated signal. Besides, the process of lowpass filtering is simple in nature and can be efficiently implemented either by software [fast 


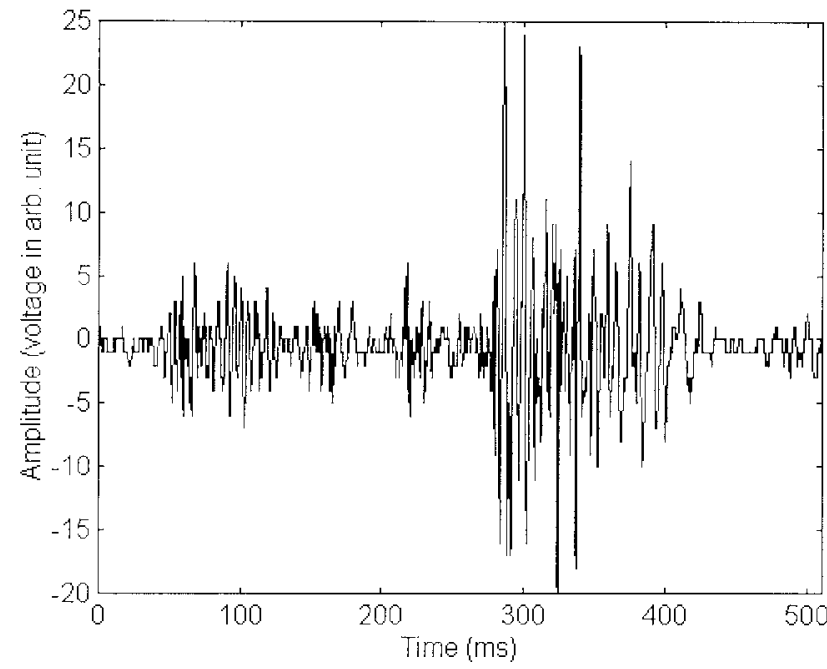

(a)

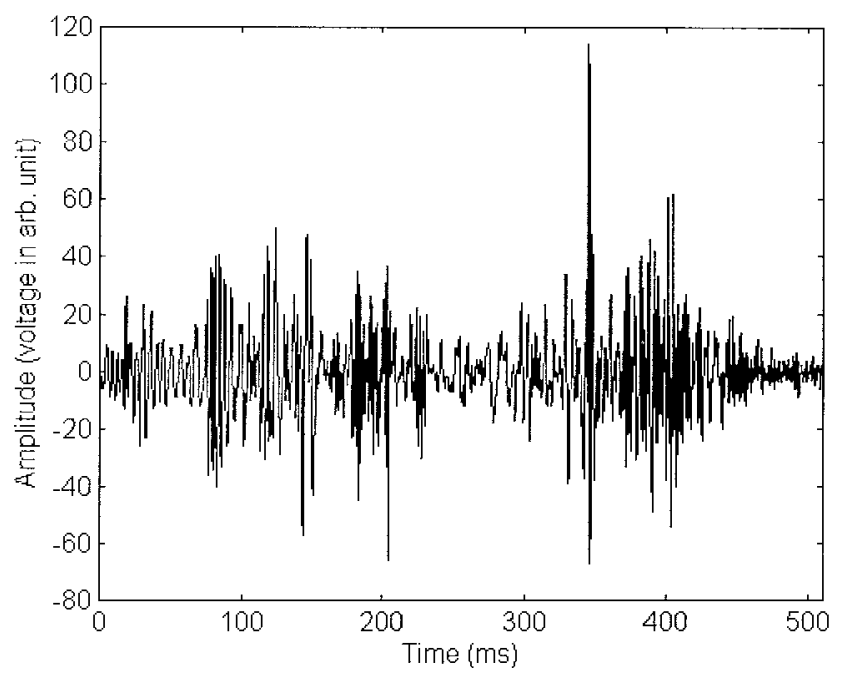

(c)

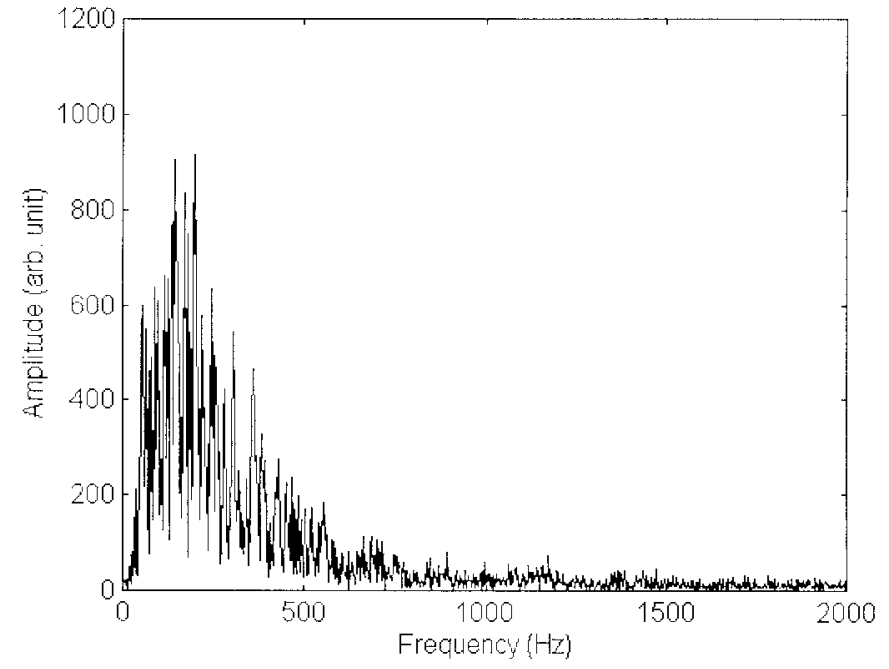

(b)

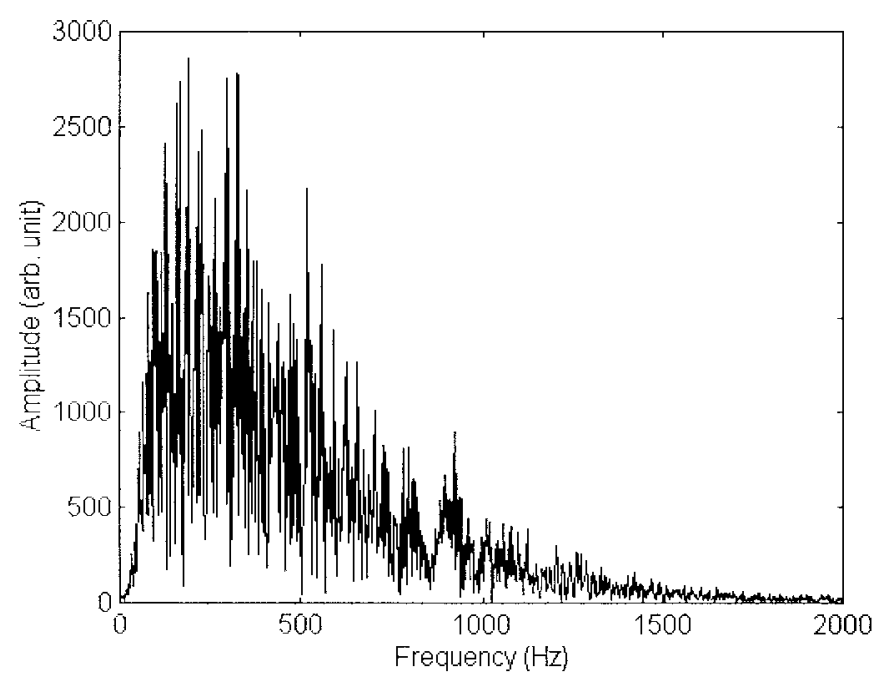

(d)

Fig. 3. Waveforms and spectra of a single cardiac cycle: (a) waveform of a normal heartbeat, (b) spectrum of (a), (c) waveform of an embolic heartbeat (with $0.05 \mathrm{ml}$ of injected air), and (d) spectrum of (c).

Fourier transform (FFT) algorithm] or hardware (digital filter) approaches. The cutoff frequency was chosen to be $3 \mathrm{~Hz}$, which is the average heart rate of dogs. The full-wave rectified DC-clipped DHS was lowpass filtered. The resultant signal envelope and its local minima, together with the original DHS, are shown in Fig. 2. Each pair of consecutive local minima indicates a logical partition of the DHS. In addition, the time length of each partition provides the period of the corresponding heartbeat. The lowpass filter actually attenuates a great deal of noise, and we can successfully partition the DHS into single beats. We also indicate in Fig. 3 the waveforms and spectra of a single cardiac cycle with and without embolic air. It is obvious that the embolic heartbeat contains additional high-frequency components as compared with the normal heartbeat.

The WT was then applied to the DHS signal. A representative example of the WT of the DHS of a dog is shown in Fig. 4. From visual inspection, the WT of the DHS at $j=1$ shows differences which would aid in distinguishing the embolic heartbeat from the normal. The embolic heartbeat was confirmed by an experienced anaesthetist by listening to the original acoustic signal. We next calculated the power of single heartbeats of the WT of the DHS at different scales. The resultant power data, which were normalized with respect to the control mean, are plotted in Fig. 5. The plot of the power data of the original DHS (i.e., $j=0$ ) is also included for comparison.

The embolic heartbeats can now be readily identified by means of thresholding as the contrast of the embolic to normal signal power is greatly enhanced. A threshold value of two times the mean control power level was experimentally found to be suitable for effective and reliable detection of embolic heartbeats (Fig. 5). The twice-baseline threshold was chosen on empirical grounds. Choosing other threshold values, e.g., three times or four times gave similar results. Using threshold values lower than two times gave a noisier signal. For each air 


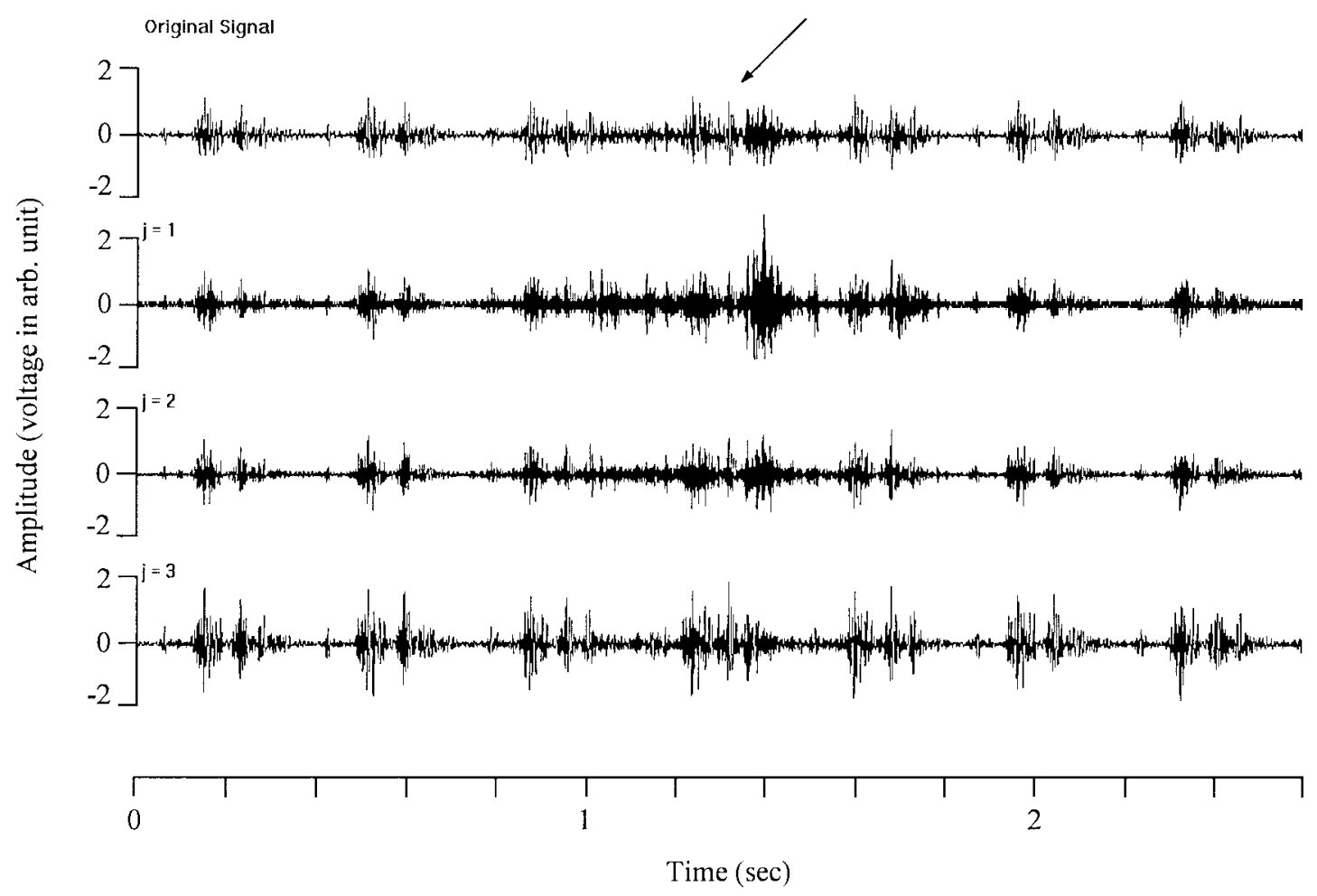

Fig. 4. A typical DHS signal containing seven heartbeats and its WT at different scales $(j=1$ to 3$)$. With a $0.02 \mathrm{ml}$ of air injected, the embolic heartbeat (marked by an arrow) was confirmed by an experienced anaesthetist by listening to the DHS signal.

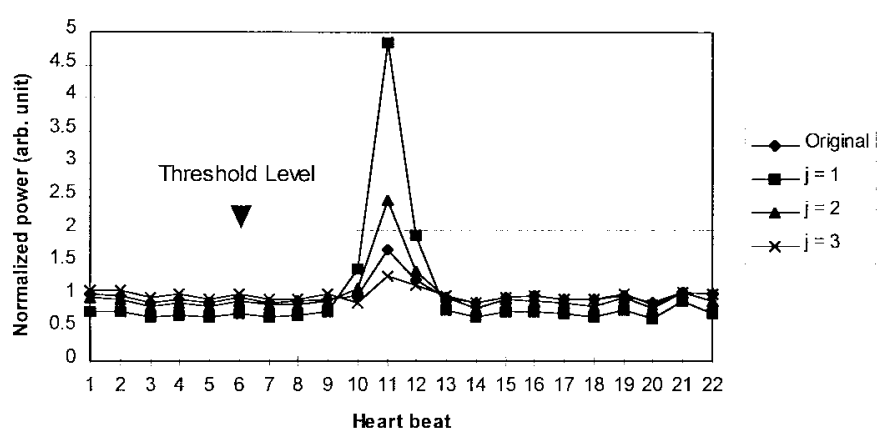

Fig. 5. Normalized power of individual heartbeats obtained after WT of a DHS containing the time segment in Fig. 4. The embolic heartbeat is identified by an increase of power (for $j=1$ and 2) above a threshold level (two times the mean power level of the control signal before air injection).

injection, we summed the normalized power of the heartbeats above the threshold and called it the "cumulative embolic power (CEP)." The correlation between the CEP and volume of injected air was examined by linear regression. The increase of the CEP above the control level was also determined. Student's $t$-test was used whenever appropriate. $P<0.05$ was considered to be significant.

\section{RESULTS}

At all scales (i.e., $j=0$ to 3 ) we tested, the correlation between the CEP and the volume of injected air was highly significant $(P<0.0005)$. In particular, the correlation coefficient reached values as high as 0.83 and 0.81 for $j=1$ and 2, respectively. Besides, the increase of the CEP above the control was significant $(P<0.01$ and $P<0.05$ for $j=1$ and 2 , respectively) at volumes as low as $0.01 \mathrm{ml}$. The relationship between the CEP and the volume of injected air at $j=1$ and 2 is detailed in Fig. 6. The average data and the error bars in Fig. 6(a) and (b) can be used for viewing inter-subject variations. Linear regressions of Fig. 6(a) and (b) were performed in the log-log scale and the results are shown in Fig. 6(c) and (d). The data of an individual subject (Dog 3) are also shown [Fig. 6(e) and (f)].

For the purpose of comparison, we processed the same datasets using our previous method [6]. Specifically, the maximum frequency, $\left(F_{\max }\right)$, was estimated for each heartbeat using the percentile-and-threshold crossing method [7]. The power of the high-frequency components above $F_{\max }$ (i.e., HF power) of each heartbeat was also computed. The $F_{\max }$ and the HF power of the heartbeats before injection were calculated to serve as controls.

We obtained significant increase $(P<0.005)$ in the singlebeat $F_{\max }$ above the control at volumes of $0.01 \mathrm{ml}$ or greater. The correlation between the greatest single-beat $F_{\max }$ and the volume of injected air was also significant $(P<0.0005, r=$ 0.64). As for the HF power, the increase of the HF power compared with control was significant $(P<0.05)$ at larger volumes (i.e., $0.02 \mathrm{ml}$ or greater). The correlation between the HF power and the volume of injected air was also significant $(P<0.0005, r=0.68)$. Table II summarizes the results obtained from the two methods. It is obvious that the WT method performs better than our previous method in that the former at $j=1$ or 2 enhances both the sensitivity and correlation with the volume of injected air. 


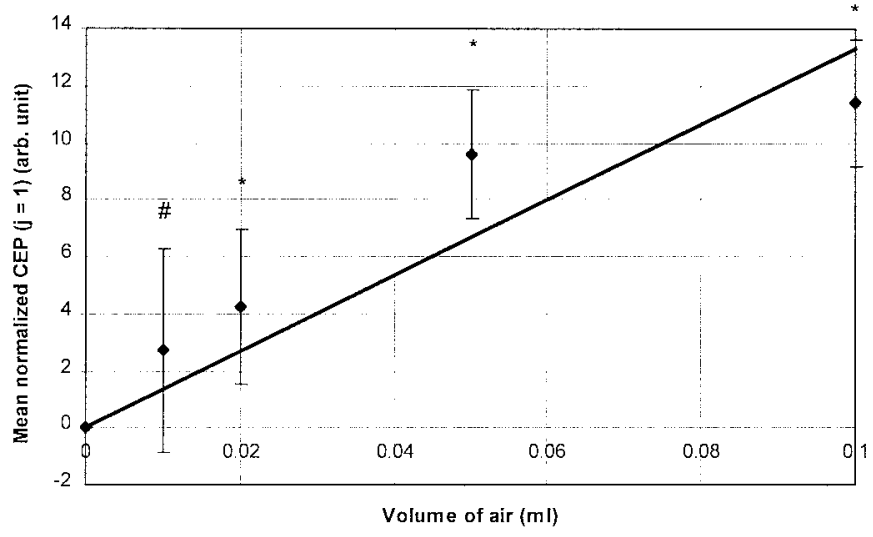

(a)

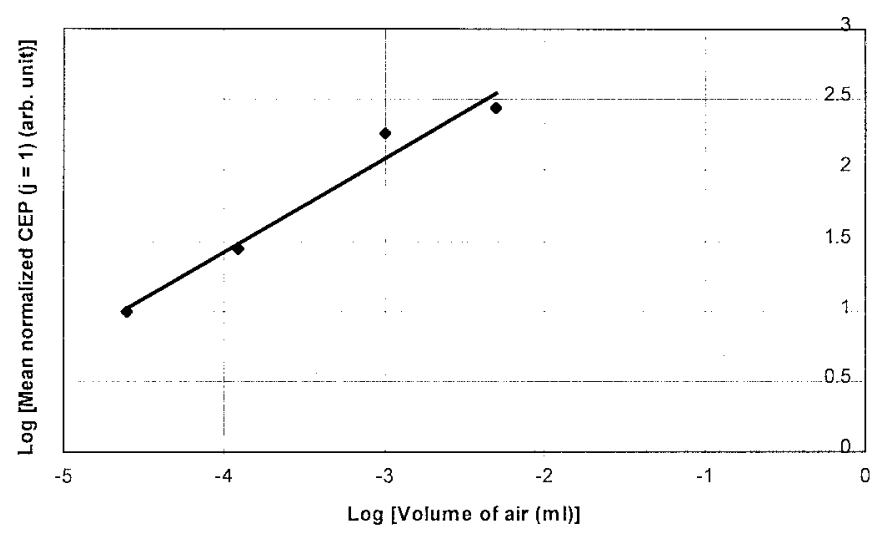

(c)

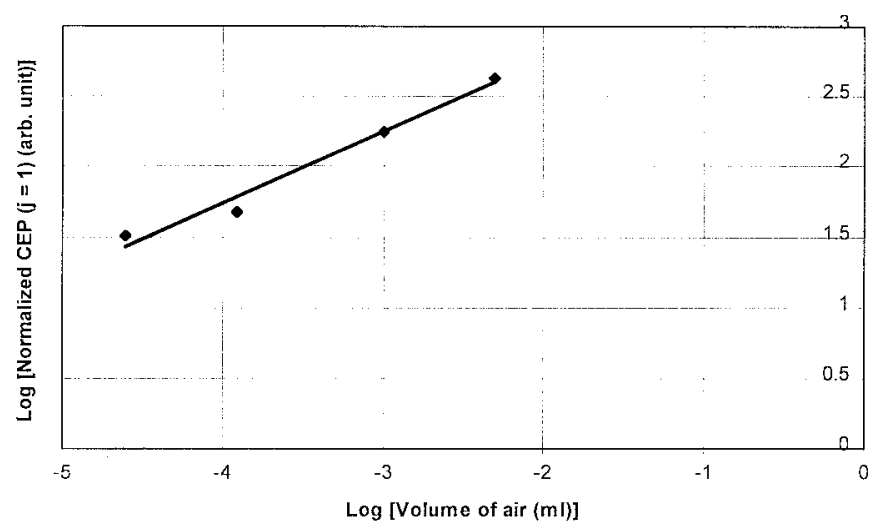

(e)

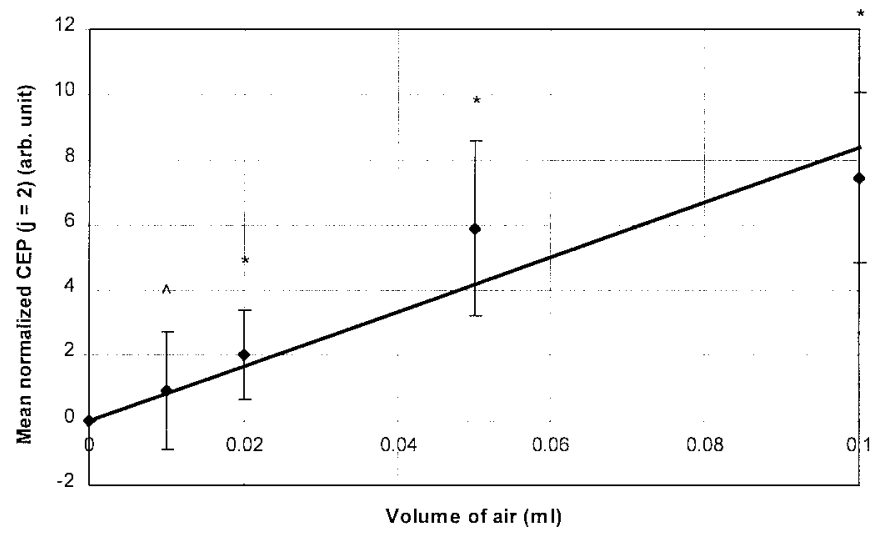

(b)

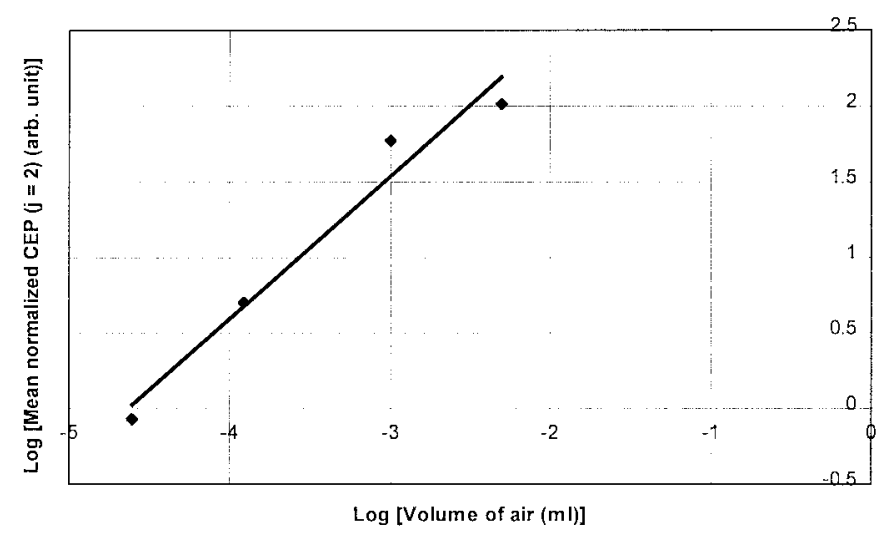

(d)

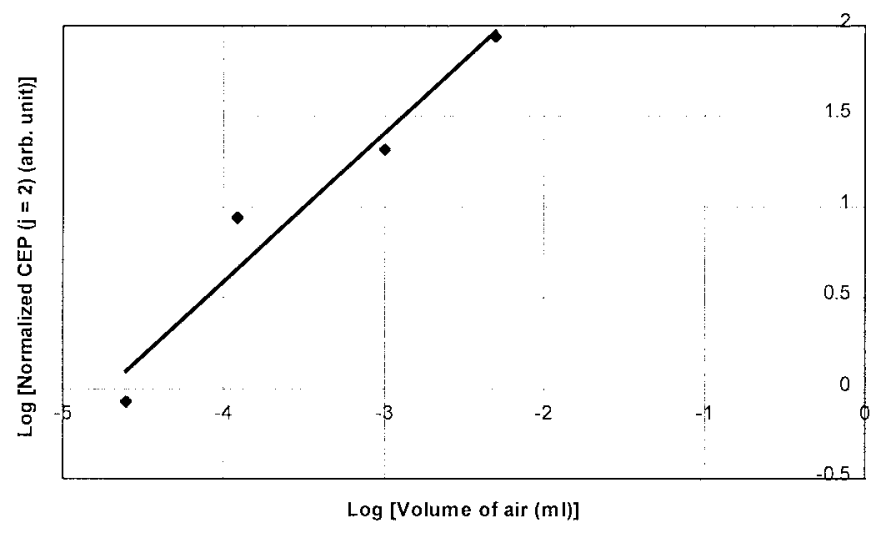

(f)

Fig. 6. Relationship between the CEP and the volume of injected air at two different $j$ values: (a) linear plot of the mean normalized CEP $(j=1)$ versus the volume of injected air $(r=0.83)$ and (b) linear plot of the mean normalized CEP $(j=2)$ versus the volume of injected air $(r=0.81)$. Each point shows the mean and standard deviation (error bar) of 12 incidences of air injections obtained in four dogs. Significant increase: ${ }^{\wedge} P<0.05$, \#P<0.01 and ${ }^{*} P<0.0005$ compared with control, (c) linear regression in log-log scale of (a) $\left(y=0.66 x+4.06, r^{2}=0.97\right)$, (d) linear regression in log-log scale of (b) $\left(y=0.94 x+4.35, r^{2}=0.96\right)$, (e) $\log -\log$ plot of the normalized CEP $(j=1)$ versus the volume of injected air for Dog $3\left(y=0.51 x+3.78, r^{2}=0.98\right)$, and (f) $\log -\log$ plot of the normalized CEP $(j=2)$ versus the volume of injected air for Dog $3\left(y=0.81 x+3.84, r^{2}=0.95\right)$. Natural $\log$ is used for all the log-log plots. Data of (e) and (f) represent averages of three air injections in Dog 3.

\section{FAST DEteCtion Algorithm FOR VENOUS AIR EMBOLISM}

\section{A. Comparison of Data Processing Speed}

A preliminary speed performance test of the present WT method and the previous time-frequency method was per- formed on an IBM PC (90 MHz Pentium) and the results are shown in Table III. It is obvious that the former is computationally much more efficient, as it boosts the data processing speed by nearly one-hundredfold of that of the latter. Hence, the WT method can facilitate real-time detection of VAE even in the absence of dedicated signal processing 
TABLE II

Comparison of the Present (WT) and Previous ( $F_{\max }$ And HF Power) Methods in Terms of Correlation and SEnsitivity

\begin{tabular}{|c|c|c|c|c|c|}
\hline \multirow[t]{2}{*}{ Method } & \multirow[t]{2}{*}{$j$} & \multicolumn{2}{|c|}{ Correlation } & \multicolumn{2}{|c|}{$\begin{array}{c}\text { Sensitivity } \\
\text { (increase compared with control) }\end{array}$} \\
\hline & & $P$ & $r$ & $P$ & Injected air (ml) \\
\hline \multirow{4}{*}{ WT } & 0 & $<0.0005$ & 0.62 & $<0.005$ & $>=0.05$ \\
\hline & 1 & $<0.0005$ & 0.83 & $<0.01$ & $>=0.01$ \\
\hline & 2 & $<0.0005$ & 0.81 & $<0.05$ & $>=0.01$ \\
\hline & 3 & $<0.0005$ & 0.55 & $<0.025$ & $>=0.05$ \\
\hline$F_{\max }$ & & $<0.0005$ & 0.64 & $<0.005$ & $>=0.01$ \\
\hline HF power & & $<0.0005$ & 0.68 & $<0.05$ & $>=0.02$ \\
\hline
\end{tabular}

TABLE III

Time of Processing Using the Present (WT) and Previous $\left(F_{\max }\right.$ AND HF Power) Methods as Measured on an IBM PC (90 MHz Pentium). Result of Averaging the Processing Times of 20 Heartbeats

\begin{tabular}{cc}
\hline Module & Time per beat $(\mathrm{ms})$ \\
\hline Lowpass filter & 294 \\
Time-frequency distribution & 4519 \\
$F_{\max }$ & 28 \\
HF power & 32 \\
WT $(j=0$ to $j=3)$ & 47 \\
Power calculation & 3 \\
\hline
\end{tabular}

chips. However, the processing time for lowpass filtering (294 ms per beat) seems to be not fast enough for real-time processing as it is comparable to the average heartbeat period of dogs (average heart rate of $\operatorname{dogs}=3 / \mathrm{s}$ ). Therefore, the procedures of lowpass filtering, WT, and power calculation should be further refined such that fast and reasonably sensitive spotting of embolic heartbeats can be achieved.

\section{B. Refinement of the Wavelet Transform}

As the occurrence of VAE is in general not very frequent during an operation, it is not computationally efficient and cost-effective to process the WT of DHS at all scales (i.e., $j=1$ to 3 ) for real-time detection of VAE. Fortunately, we discovered that the WT of the DHS at the scale $2^{1}$ provides good sensitivity and significant correlation with the volume of embolic air. In addition, we can exploit some features of this scale of the WT as well as the wavelet function itself to increase the data processing speed. Having spotted an occurrence of VAE, we can conduct a more thorough quantitative analysis of the EHS so as to obtain an estimate of the volume of embolic air.

Our refinement of the WT starts with (17): for $j=1$, it can be simplified to

$$
W_{2} f(x)=2(f(x-1)-f(x)), \quad x \in Z
$$

as the impulse response of $G(\omega)$ is $(-2,2)$ (Table I). With a constant scaling of one-half of (19), it can be further reduced to

$$
W_{2} f(x)=f(x-1)-f(x)
$$

Hence, the complexity of our revised WT algorithm is merely $O(N)$ with unit complexity constant. As our digitized DHS signals are all integers, only integer manipulation is needed.
In fact, for $N$ points (integers) of a digitized DHS signal, our algorithm only requires $N$ subtractions of integers while no multiplication or division is needed.

\section{Combination of Lowpass Filtering and Power Calculation}

The lowpass-filtering step can be replaced by the movingaverage procedure [14] as the former is essentially a kind of averaging. Embedding the power calculation in the moving average results in a "moving square average (MSA)," which is defined by

$$
\operatorname{MSA}(x)=\frac{1}{L+M+1} \sum_{k=-L}^{M} f^{2}(x-k) .
$$

With a constant scaling of $L+M+1$ (i.e., the window length), (21) can be reduced to

$$
\operatorname{MSA}(x)=\sum_{k=-L}^{M} f^{2}(x-k) .
$$

The window length we use is 1024 points with $L=511$ and $M=512$ (i.e., $256 \mathrm{~ms}$ at $4-\mathrm{kHz}$ sampling frequency, which is close to the average cardiac period of dogs). For $N$ signal points, we need $1024 N$ multiplications and 1023 $N$ additions with direct implementation using (22), and the complexity constant of $O(N)$ is equal to the window length. In order to reduce the magnitude of the complexity constant, we propose the following.

Assume that we need to compute $\operatorname{MSA}(x)$ from $x=t$ to $x=t+N-1$ (i.e., $N$ points). We first calculate $\operatorname{MSA}(t)$ which requires 1024 multiplications and 1023 additions. Then, $\operatorname{MSA}(t+1)$ can be computed as follows:

$$
\operatorname{MSA}(t+1)=\operatorname{MSA}(t)+f^{2}(t+512)-f^{2}(t-512) .
$$

Changing the variable in (23) as $x=t+1$, we obtain a general equation for computing $\operatorname{MSA}(x)$ as

$$
\operatorname{MSA}(x)=\operatorname{MSA}(x-1)+f^{2}(x+511)-f^{2}(x-513) .
$$

Hence, we need two multiplications, one addition, and one subtraction for each signal point that follows. For $N$ signal points as a whole, we need $2 N+1022$ multiplications, $N+1022$ additions, and $N-1$ subtractions. Allowing for a fixed overhead which depends on the window length, we reduce the complexity constant from 1024 to 2 .

The MSA's of the original DHS signal and the WT of DHS at $j=1$ (as in Fig. 4) are shown in Fig. 7. The enhancement of the contrast of the embolic to normal signal power is obvious. The spotting of the embolic heartbeats can thus be achieved by the same thresholding technique we adopted previously. The complexity of this thresholding procedure is again $O(N)$ with unit complexity constant. A final speed performance test on the refined algorithm (i.e., WT at $j=1$, MSA, and thresholding) showed that, on the average, only $8 \mathrm{~ms}$ is needed for processing a single beat. 


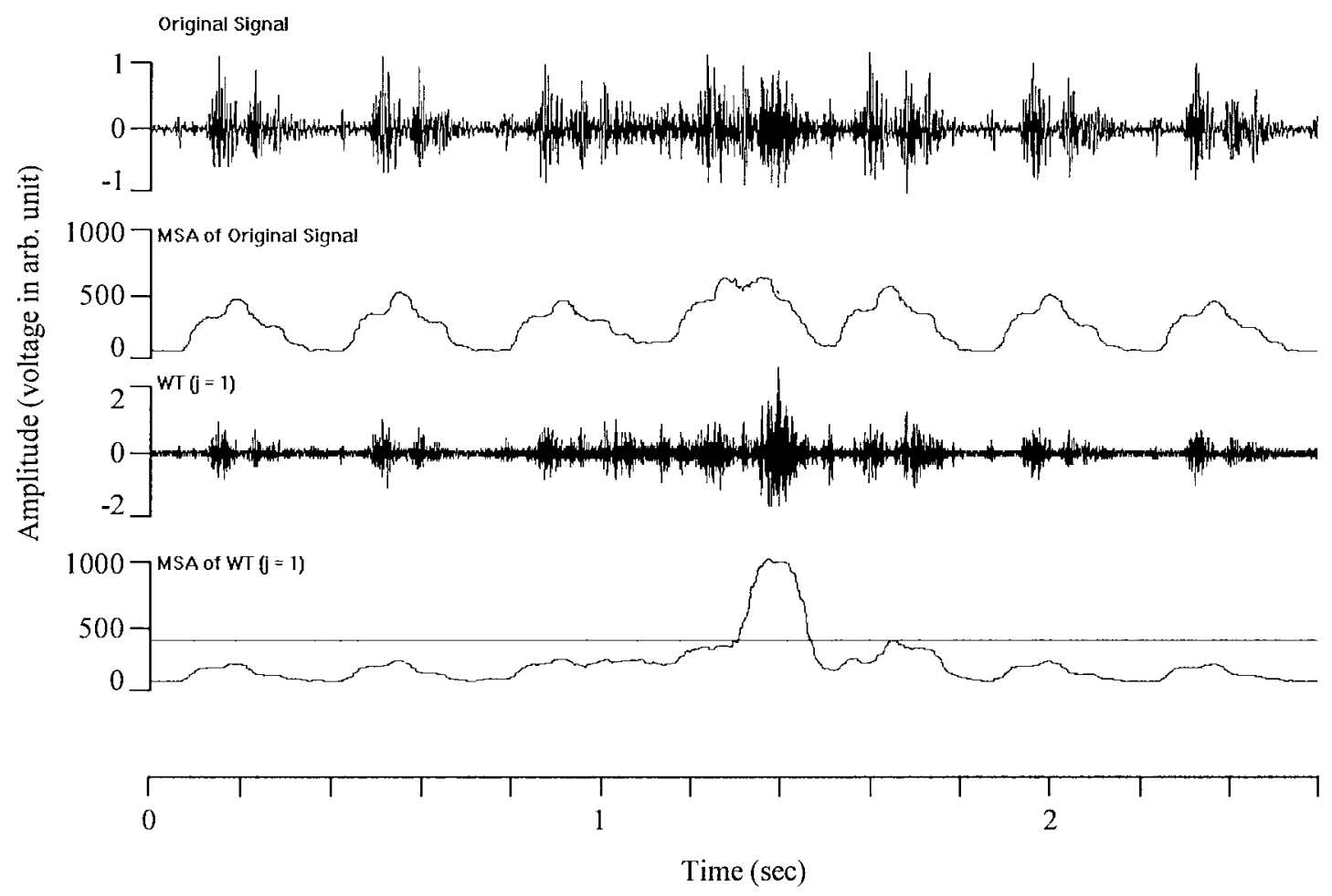

Fig. 7. MSA of the DHS signal (same as Fig. 4) and MSA of its WT at $j=1$. The embolic heartbeat is detected by the MSA signal crossing the threshold level (horizontal line or two times the mean MSA of the control) in the WT (bottom tracing).

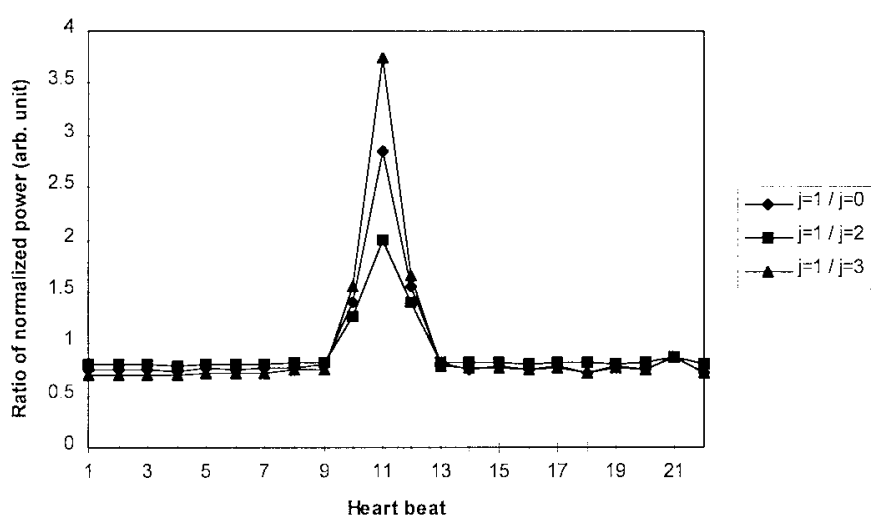

Fig. 8. Plot of the ratio of normalized power in Fig. 5. Similar results are obtained but likely with increased robustness in a noisy environment.

\section{DISCUSSION AND CONCLUSION}

The WT of the DHS provides not only a fast detection of VAE, but also an accurate estimation of the volume of embolic air. The WT of the DHS at $j=1$ can be implemented very efficiently $(O(N)$ complexity with unit complexity constant) by exploiting the antisymmetric two-coefficient impulse response of the highpass filter $G(\omega)$, while the lowpass filtering and power calculation steps can be combined into a fast MSA procedure $(O(N)$ complexity with complexity constant equal to two). The WT also provides good sensitivity $(P<0.01$ at $0.01 \mathrm{ml}$ of injected air) and significant correlation $(P<$ $0.0005, r=0.83$ ) between the CEP and the volume of injected

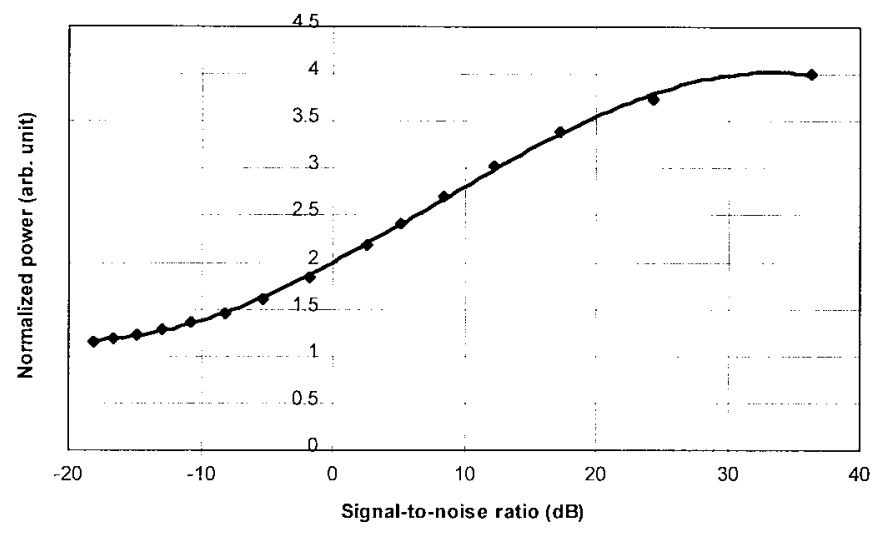

Fig. 9. Relationship between the SNR and the normalized power of the WT $(j=1)$ of the embolic heartbeat of Dog 3 with $0.02 \mathrm{ml}$ of air injected. Cubic polynomial fit: $y=-0.00004 x^{3}+0.0009 x^{2}+0.0755 x+1.9984$.

air so that detailed quantitative analysis of the embolic air can be achieved at a later stage. Although our VAE experiment was carried out on dogs, similar results for humans are expected as they would share similar physiological conditions. As the heart rate of dogs is higher than that of adult humans, the overall processing speed of $8 \mathrm{~ms}$ per beat for dogs shows the feasibility of the implementation of a real-time monitoring system for VAE in humans.

In general, the complexity of our present algorithm is $O(N)$ with unit complexity constant involving computation of integers. This is an improvement regarding computational 


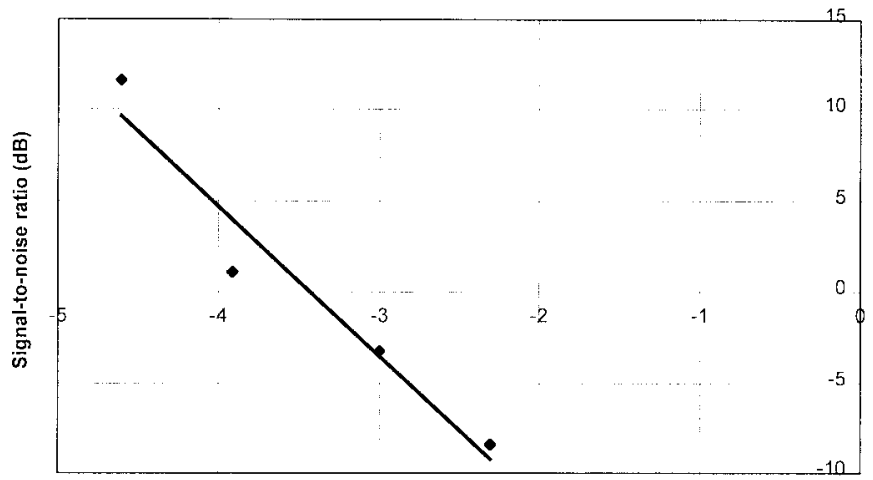

Log [Volume of air $(\mathrm{ml})]$

Fig. 10. Relationship between the minimum SNR at which the EHS can be detected and the natural $\log$ of the volume of injected air for Dog 3. Linear fit: $y=-8.20 x-28.05$.

speed over our previous method, the complexity of which (i.e., the FFT algorithm) is $O(N \log N)$ with a complexity constant of greater than one [14] in addition to complex floating point calculations.

The EHS contains high-frequency components not present in the normal heart sounds. These high-frequency components are probably due to the highly reflective and rapidly moving air bubbles as the Doppler ultrasound instrument presents the audible heart sound with frequency proportional to the velocity of the reflective structures. Since the high-frequency components appear in more-or-less the same frequency bands across different dogs, we take that as an indication of the similarity of traveling air bubbles across individual animals.

Both the previous and present methods make use of the property that the EHS contains extra high-frequency components. The single cutoff frequency employed in the previous method may be too crude and may hide certain important details in the high-frequency region which are valuable for an accurate estimation of the volume of embolic air. The same thing happens when a standard bandpass filter is applied to the DHS signal. Optimal linear filter approaches such as Wiener or matched filtering should also be a good choice, but which would have greater computational cost. On the other hand, the WT can quickly scan the DHS signal at different scales, which is similar to bandpass filtering the signal at successively narrower bandwidths, so that finer details (i.e., the transient changes) of the signal can be revealed. Besides the feasibility of a real-time implementation of the WT, DHS signals obtained at different scales could provide additional features for diagnosing various kinds of emboli in the blood. We need to point out that the optimal filter for the DHS signal of a given dog could be different from that for others, due to the variable anatomy (e.g., of the chest wall), and the difference in placement of the probe. The choice of the WT would allow fast adjustment to obtain optimal filter settings. As both the present and previous methods analyze the highfrequency components of the EHS, it is not surprising that they arrive at similar results, though our present method definitely performs better in terms of speed, sensitivity, and correlation.
Doppler ultrasonic instruments are normally equipped with effective shielding mechanism that suppresses radio-frequency interference. Battery operation is often possible, thereby eliminating the noise picked up from the power line. Except for short noisy periods due to electrocautery during skin incision or coagulation at the beginning of the operation, DHS signals in the operating room are often clear and clean. As a result, we could use the absolute power of the signal after the $j=1$ filter for the detection of VAE. However, in those cases of persistent noisy DHS signals, the ratio of the power of the signal at some combinations of the $j=1,2$, and 3 filters (Fig. 8) should be used as they might be more robust in a noisy environment. The additional complexity of calculating these ratios is not computationally intensive and real-time processing may still be feasible. We also studied the performance of our present method with different intensities of additive white Gaussian noise on the DHS. In general, the enhancement of the contrast of the embolic to normal signal power decreases with increasing noise intensity [i.e., decreasing signal-to-noise ratio (SNR)]. Fig. 9 shows that the enhancement suffers minor degradation at SNR above $20 \mathrm{~dB}$. Then, it decreases linearly from $20 \mathrm{~dB}$ down to $-10 \mathrm{~dB}$ and gradually approaches unity (i.e., no enhancement) below $-10 \mathrm{~dB}$. At twice-baseline threshold (i.e., normalized power equals two), the minimum SNR at which the EHS can be detected is around $0 \mathrm{~dB}$. This minimum SNR decreases with increasing volume of injected air (Fig. 10).

\section{REFERENCES}

[1] J. D. Michenfelder, R. H. Miller, and G. A. Gronert, "Evaluation of an ultrasonic device (Doppler) for the diagnosis of venous air embolism," Anesthesiol., vol. 36, pp. 164-167, 1972.

[2] A. M. Malinow, J. S. Naulty, C. O. Hunt, S. Datta, and G. W. Ostheimer, "Precordial ultrasonic monitoring during cesarean delivery," Anesthesiol., vol. 66, pp. 816-819, 1987.

[3] J. B. English, D. Westenskow, M. R. Hodges, and T. H. Stanley, "Comparison of venous air embolism monitoring methods in supine dogs," Anesthesiol., vol. 48, pp. 425-429, 1978.

[4] R. F. Cucchiara, M. Nugent, and J. B. Seward, "Air embolism in upright neurosurgical patients: Detection and localization by two-dimensional transesophageal echocardiography," Anesthesiol., vol. 60, p. 353, 1984.

[5] R. C. Shupak, "Air embolism and its influence on anesthetic management," Current Rev. Clin. Anesthesia, vol. 11, pp. 113-124, 1991.

[6] P. W. Lui, Y. M. Lin, F. H. Y. Chan, M. Y. Tsou, S. Wang, F. K. Lam, and P. W. F. Poon, "Spectral characteristics of embolic heart sounds detected by precordial Doppler ultrasound during venous air embolism in dogs," Br. J. Anaesthesia, vol. 71, pp. 689-695, 1993.

[7] L. Y. L. Mo, L. C. M. Yun, and R. S. C. Cobbold, "Comparison of four digital maximum frequency estimators for Doppler ultrasound," Ultrasound Med., Biol., vol. 14, pp. 355-363, 1988.

[8] H. L. Alder and E. B. Roessler, Introduction to Probability and Statistics. San Francisco, CA: Freeman, 1972, ch. 10, pp. 153-166.

[9] B. C. B. Chan, F. H. Y. Chan, F. K. Lam, P. W. Lui, and P. W. F. Poon, "Real-time monitoring of venous air embolism with Doppler heart sound using multiresolution wavelet analysis," in Engineering and Physics in Medicine, Conf. Program, Queenstown, New Zealand, Nov. 1995, p. 185 .

[10] O. Rioul and M. Vetterli, "Wavelets and signal processing," IEEE Signal Processing Mag., pp. 15-38, Oct. 1991.

[11] Y. Meyer, Wavelets and Operators. Cambridge, U.K.: Cambridge Univ. Press, 1992.

[12] I. Daubechies, Ten Lectures on Wavelets. Philadelphia, PA: SIAM, 1992.

[13] S. Mallat and S. Zhong, "Characterization of signals from multiscale edges," IEEE Trans. Pattern Anal. Machine Intell., vol. 14, pp. 710-732, 1992.

[14] A. V. Oppenheim and R. W. Schafer, Discrete-Time Signal Processing. Englewood Cliffs, NJ: Prentice-Hall, 1989. 


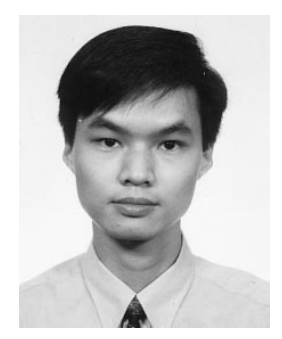

Brent C. B. Chan (S'95) was born in Hong Kong in 1970. He received the Bachelor of Engineering (First Class Honors) degree in 1992, and is now pursuing the Ph.D. degree, both in electrical and electronic engineering at the University of Hong Kong.

He worked for more than two years in industries before joining the Ph.D. program. His research interests include biomedical engineering, medical signal processing, and real-time processing.

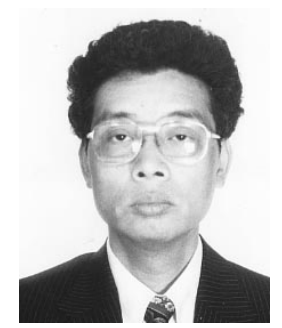

Francis H. Y. Chan (M'80) was awarded the Ph.D. degree in electrical engineering in 1975 from Bristol University, Bristol, U.K.

He worked for more than four years in British industries before joining the Electrical and Electronic Engineering Department, the University of Hong Kong in 1976. Currently, he is Professor and the Director of the University of Hong Kong Biomedical Engineering Centre, coordinating biomedical engineering research in the university. His current research interests are in biomedical engineering and medical signal processing.

Dr. Chan is a Chartered Engineer, and Fellow of both the Institution of Electrical Engineers (IEE) and Hong Kong Institution of Engineers.

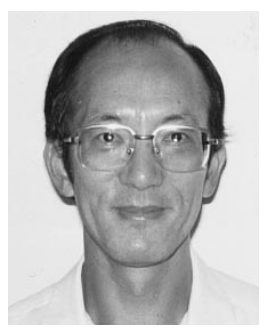

F. K. Lam obtained the B.Sc.(Eng.) degree from the University of Hong Kong, and the M.Sc.and Ph.D. degrees from Loughborough University, Leics, U.K.

After a few years working in Britain, he returned to Hong Kong University in 1970 and is currently a Senior Lecturer in the Department of Electrical and Electronic Engineering. His areas of specialization are in signal processing and communications.

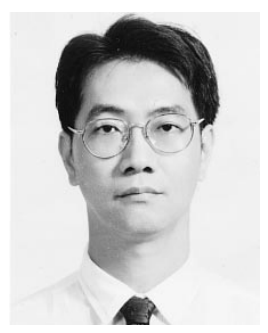

Ping-Wing Lui received the Bachelor of Medicine degree in 1981 from the National Defense Medical Center of Taiwan. He was also awarded the Ph.D degree in medical science in 1989 from the National Yang-Ming University, Taiwan

Since then, he has been working as an anesthesiologist in Veterans General Hospital, Taipei, Taiwan. Currently, he is the head of Neuroanesthesia and Associate Professor of Anesthesiology in the Department of Anesthesiology, School of Medicine, National Yang-Ming University, Taiwan. His major research interests include monitoring in neurosurgical anesthesia, neuropharmacology of muscular rigidity induced by opiates, and mechanism and clinical management of chronic pain.

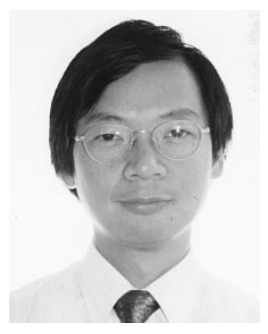

Paul W. F. Poon received the B.Sc. degree in biology from the Chinese University of Hong Kong, the M.Phil. degree (physiology) from the University of Hong Kong, and the Ph.D. degree (neuroscience) from the Indiana University, Bloomington, in 1972, 1974 , and 1979, respectively.

From 1979 to 1994, he was Lecturer in the Department of Physiology, Faculty of Medicine, University of Hong Kong. Currently, he is Professor in the Department of Physiology, School of Medicine, National Cheng Kung University, Tainan, Taiwan.

Dr. Poon is member of Chinese Biomedical Engineering Society, American Physiological Society, Chinese Neuroscience Society, and Association for Research in Otolaryngology. His research interests include neural coding of complex sounds, auditory physiology, and biomedical signal processing. 